\title{
Internet und Geschlechterordnung: Expertinnen im Gespräch
}

\author{
Johanna Dorer
}

Obgleich der Frauenanteil im Internet rasant ansteigt, bedeutet das noch keineswegs, dass damit auch der Geschlechterdualismus und seine hierarchischen Strukturen an Bedeutung verlieren würden. Sowohl mediale als auch gesellschaftliche Rabmenbedingungen geben einen Internet-Diskurs vor, der in seiner geschlechterbinären Codierung auf die Anwendungspraxis und die darin ausgedrückte Selbstpositionierung als männlich oder weiblich Einfluss hat. Interviews, die mit 23 Internet-Expertinnen in Österreich gefübrt wurden, zeigen, dass es den Frauen im derzeit noch männlich dominierten Berufsfeld Internet nur sebr eingeschränkt gelingt, sich an der Neuformulierung von Geschlechtergrenzen zu beteiligen. Entgegen den Vorstellungen und Utopien von Cyberfeministinnen, Frauen könnten durch eine aktive Auseinandersetzung mit dem Internet Geschlechtergrenzen aufheben, verweist vorliegende qualitative Studie auf die subtil wirksamen Mechanismen und Praktiken der Geschlechterpositionierung und das alltägliche „doing gender".

\section{Einleitung}

Noch vor einigen Jahren galt das Internet als eine reine Männerdomäne. Für Frauen war der Zugang zur neuen Kommunikationstechnologie schwierig, und der Frauenanteil erreichte kaum mehr als 10 Prozent. (Dorer 1997, 20) Dies hat sich mittlerweile geändert. In den Vereinigten Staaten gibt es seit Mitte 2000 bereits ein ausgeglichenes Verhältnis von Internetnutzern und Internetnutzerinnen, in Österreich betrug zur gleichen Zeit der Frauenanteil 38 Prozent (N.N. 2000, ORF 2000)ํ. Geht die Entwicklung in dieser Geschwindigkeit voran, so ist in den meisten europäischen Ländern spätestens in vier Jahren mit einem ausgewogenen Geschlechterverhältnis in der Zugangsstatistik zu rechnen.

Damit ist aber keineswegs die Wirksamkeit der Geschlechterordnung außer Kraft gesetzt. Denn Gender als soziales und kulturelles Konstrukt wirkt in seiner Gesamtheit auf die Vorstellungen und Erwartungen, die in einer Gesellschaft in Bezug auf Männlichkeit und Weiblichkeit existieren. Geschlecht ist dabei nicht eine soziale Rolle, sondern Ergebnis einer Vielzahl an Repräsentationen einerseits und diskursiver Praktiken andererseits, bzw. wie de Lauretis $(1996,68)$ formuliert: „Die Konstruktion des Geschlechts ist sowohl Produkt als auch Prozess von Repräsentation und Selbstrepräsentation“. Das bedeutet, dass Geschlecht als soziales Arrangement in verschiedenen Alltagspraktiken immer wieder neu - in Form eines „doing gender“ - produziert wird, und die Selbstpositionierung bzw. Selbstrepräsentation als Mann oder als Frau die Aneignung der entsprechenden Bedeutungen beinhaltet.

Diese Selbstpositionierung/Selbstrepräsentation wird bestimmt von einer „ganzen Batterie an Repräsentationen“. (Cockburn/Ormrod 1997, 25) Beginnend bei medialen und werblichen Bildern, die in ihrer stereotypenbildenden Funktion Entwürfe für Männ-

1 Für das Jahr zuvor weist für Deutschland die Online-Studie 1999 einen Frauenanteil von 35 Prozent aus. Vgl. ARD/ZDF-Arbeitsgruppe Multimedia (1999). 
lichkeit und Weiblichkeit bereitstellen, über gesellschaftliche Institutionen wie Familie, Ausbildungsstätten, Gerichte etc., bis zu sämtlichen sozialen, politischen und ökonomischen Prozessen werden ständig Geschlechterdiskurse in Form von symbolischen Repräsentationen hervorgebracht. Medien spielen bei der Konstruktion kultureller Konzeptionen für Männlichkeit und Weiblichkeit eine immer wichtiger werdende Rolle.

Die Einschreibung von Geschlecht in die neue Technologie erfolgt auf unterschiedlichen Ebenen. Im Folgenden geht es nun um die Frage, wie das Internet als neue Technologie an der Geschlechterpositionierung beteiligt ist. Mediale Repräsentationen, gesellschaftliche Rahmenbedingungen und die Alltagspraxis des „doing gender“ werden dabei in den Blick genommen. Den Ausgangspunkt bilden einerseits ein Dreistufen-Modell medialer Repräsentationen von Geschlecht in Bezug auf die neue Technologie und andererseits Überlegungen zu geschlechtlich codierten Rahmenbedingungen des Internet. Anschließend werden anhand der Ergebnisse einer qualitativen Befragung von Internet-Expertinnen die Konstruktionsmechanismen des „doing gender" nachgezeichnet. Bei dieser Analyse geht es um die Frage, wie sich die Internet-Expertinnen in Bezug auf eine männlich codierte Medientechnik in einem männlich dominierten $\mathrm{Be}-$ rufsfeld geschlechtlich positionieren und wie ihr Handeln in Geschlechterdiskurse eingeschrieben ist.

\section{Netzentwicklung und mediale Repräsentationen von Internet und Geschlecht}

Die medialen Repräsentationen von Internet und Geschlecht verlaufen nach wie vor entlang der traditionellen Geschlechterlinie. Zunehmend kommt es aber zu einer Ausdifferenzierung der medialen Konstruktionen von Männlichkeits- und Weiblichkeitsbildern. Dies lässt sich in einem Dreistufen-Modell anhand der Genealogie des Netzes nachvollziehen. Lovink/Schultz $(1999,299-310)$ unterscheiden drei Phasen der Netzentwicklung wie folgt:

- Die erste Phase (1969-1989) ist geprägt durch die Vernetzung von Großrechnern in Militär, Wissenschaft und Großunternehmen. Dies ist auch die Zeit, in der sich Jugendliche das technische "Geheimwissen“ aneignen und sich als so genannte „Hacker“ und „Cyberpunks“ für die Öffnung des Netzes einsetzen. Die Idee der virtuellen Gemeinschaft wird in zahlreichen Netzwerken praktiziert. In dieser Zeit entstanden die von der Wissenschaft - auch von den Cyberfeministinnen - euphorisch kommentierten „Mysterien“ des Internets wie Entkörperung, Unsterblichkeit, Hybridisierung von Körper und Maschine, Cyborg und Cybersex.

- Die zweite Phase (1990-1995) ist die Zeit der Mythenbildung, der Gerüchte und Erwartungen, in der die alten Medien den Mythos Internet, den Glauben an universelle Möglichkeiten verbreiten und die „digitale Revolution“ ausrufen. Es ist die Zeit der schnellen Profite, Netzutopien, der Netzkritik und der Medienkunst, wo aus so unterschiedlichen Interessen eine neue „virtuelle Klasse“ (Kroker/Weinstein 1994) hervorgeht.

- Die dritte Phase ist gekennzeichnet durch die Entwicklung des Internets hin zum Massenmedium. Die Anzahl der Nutzer/innen steigt rasch an, Klickraten und Userstatistiken und die Spuren, die Anwender/innen in unzähligen Logfiles hinterlassen, werden marktrelevant verwertet. Die digitale Vernetzung beschleunigt Globalisierungs- und Konzentrationsprozesse, verändert Geld- und Aktienmärkte, die neue Ökonomie wird zur wichtigsten Wachstumsbranche. Das Streben nach politischer Einflussnahme äußert sich - unter Bezugnahme auf das Thema Kinderpornografie in der Diskussion um Kontrolle, Reglementierung, Zensur versus Meinungs- und 
Medienfreiheit. Der hegemoniale Kampf wird nicht zuletzt auf dem Gebiet der Festlegung von Standards ausgetragen. Denn jede Nutzer/innengruppe hat ihre eigenen Standards und Benutzungsregeln entwickelt und dem Netz eingeschrieben.

Die zeitliche Systematisierung der Netzentwicklung lässt sich nun auf ein DreistufenModell der medialen Repräsentation von Internet und Geschlecht erweitern. Basis dieses Modells sind meine eigenen mehrjährigen Medienbeobachtungen, welche noch empirisch zu prüfen wären.

Den Anfängen der Netzentwicklung wurde kaum die Aufmerksamkeit der breiten Öffentlichkeit zuteil. Erst in der zweiten Phase waren es vor allem die populären Medien, die den Mythos Internet öffentlichkeitswirksam inszenierten. Dabei zeigt die mediale Repräsentation von Internet, wie die Vereinnahmung durch eine männliche Codierung den Mainstream der Bedeutungszuweisung beherrscht.

Die Themen des öffentlichen Diskurses sind uneingeschränkt männlich codiert: Technik, Cyber-Sex, Cyber-Nazismus, Cyber-Kriminalität, Hacker-Kulturen. Die Koppelung von Internet und Männlichkeit wird in der öffentlichen Debatte durch ein ausnahmsloses Auftreten von männlichen Akteuren - sowohl als Bewohner der Cyberwelt als auch als Experten - zusätzlich unterstrichen. Die mediale Konstruktion des Internet als männlicher (Herrschafts-)Raum orientiert sich dabei an der Konstruktion von männlich codierter Technik. Durch die Verbindung von Technik und Internet werden nicht nur weitere männlich codierte Phantasieräume von Allmacht und Kontrolle aktiviert, sondern gleichzeitig wird auf der Bedeutungsebene das Weibliche als das Andere hergestellt und ausgeschlossen.

Der Ausschluss von Frauen erfolgt dabei nicht nur über die Themen (Technik/Expertentum, Sex/Gewalt, Allmacht/Kontrolle), die Entstehungsgeschichte (Militär, Wissenschaft) und den Internet-Jargon, sondern auch über die Nichtthematisierung der Beteiligung von Frauen an der Entwicklung von Hard- und insbesondere der Software. ${ }^{2}$ Die (alten) Medien greifen dabei die wirkungsmächtige Gleichsetzung von Technik und Internet auf, bei welcher Männlichkeit mit Technikinteresse, Technikkompetenz und das Weibliche mit Technikscheu, Technikangst, Technikablehnung bis hin zur Technikinkompetenz verbunden wird. ${ }^{3}$ Die beiden in den Medien konstruierten Männlichkeitsbilder sind dabei auf die Bedeutungsfelder von Technik/Expertentum einerseits und Sex/Gewalt andererseits fokussiert und entsprechen damit konservativen Vorstellungen von Männlichkeit.

In der dritten Phase, der Phase der Kommerzialisierung des Internet, richten sich die Medien nicht mehr ausschließlich an männliche Internetanwender, sondern schließen zunehmend Frauen mit ein. Die mediale Repräsentation von Internet und Geschlecht führt dabei einerseits zu einer zunehmenden Ausdifferenzierung von Männlichkeitsbildern und andererseits zur Einführung eines konservativen Frauenstereotyps. Der Rahmen für letzteres wird durch die Privatsphäre und die vermeintlichen „natürlichen, weiblichen" Eigenschaften abgesteckt. Die Medien konstruieren dabei ein Weiblichkeitsbild, das die Frau auf Mutter, Köchin und Konsumentin reduziert und weit konservativer als jenes in TV-Soaps ist: Es ist die Internet-Surferin, die an Kinderbetreuung, Kochrezepten und Versandhausangeboten interessiert ist. Diese konservative

2 Vgl. dazu u. a.: Plant (1997), Haraway (1990), Hoffmann (1987), Herner (2000).

3 Die Verbindung Technik-Internet-Männlichkeit habe ich anderorts ausführlicher dargestellt, vgl. Dorer (2001). 
mediale Repräsentation von Frauen und Internet dominiert derzeit noch die Berichterstattung.

Erst sehr vereinzelt kommen in den Medien neue Frauenstereotypen, wie etwa die wenig qualifizierte Tele(heim)arbeiterin oder die Multimedia-Powerfrau vor. Die Multimedia-Expertinnen werden vom Frauen-Magazin „Mona Lisa“ als „Internetqueens“ (Makivic 2000) gefeiert und als Ausnahme von der Norm präsentiert. Harte Arbeit und Kinderlosigkeit sind dabei die nennenswerten Voraussetzungen für Erfolg. (Pfeiffer 2000)

Es ist davon auszugehen, dass sich die mediale Konstruktion von Internet und Geschlecht primär entlang zweier geschlechtlich codierter Dualismen entwickelt: Technikkompetenz versus Technikinkompetenz einerseits und Öffentlichkeit versus Privatheit andererseits. In dieser Verbindung werden verschiedene Facetten der Unterscheidung möglich:

- der technikbegeisterte Hacker - die Internet-Verweigerin,

- der berufliche Anwender - die private Surferin,

- der Internet(Multimedia-, IT-)experte - die Tele(heim)arbeiterin,

- der männliche Chatter und MUDs-Spieler - die weibliche E-Mail-Schreiberin etc.

Auf diese Weise arbeiten die (alten) Medien massiv an der gesellschaftlichen Konstruktion von Internet und Geschlechter-Differenz und -Differenzierung mit.

\section{Internet und Geschlechterpositionierung}

Internet als neue Kommunikationstechnologie trifft in vielfältiger Weise auf die in der Gesellschaft bereits vorhandenen, geschlechtlich codierten Rahmenbedingungen, die ihrerseits wieder auf die geschlechtliche Codierung der Technologie rückwirken. Denn Anfang der neunziger Jahre begann sich das Internet und Intranet sehr rasch in verschiedenen Wirtschaftsbereichen - und damit Arbeitsbereichen - zu etablieren. Aus diesem Grund waren Berufstätige früher als Nicht-Berufstätige mit der neuen Technologie vertraut, Berufstätige in höheren Positionen früher als Berufstätige mit geringerer Ausbildung und Qualifikation. Die Art der Berufstätigkeit, die gerade durch die Geschlechterzugehörigkeit bestimmt ist, konstituiert einen geschlechterdifferenten Zugang zum Internet.

Damit zusammenhängend haben die gesellschaftlich vorhandenen, geschlechtlich codierten Felder und Grenzlinien Auswirkungen auf die Alltagspraxis der Anwendung. Der Prozess des „doing gender“ vollzieht sich unter diesen Rahmenbedingungen als eine diskursive Praxis, die zumindest potenziell unterschiedliche Subjekt- bzw. Geschlechterpositionierungen zulässt. Der Prozess der Selbstpositionierung ist in der konkreten Anwendungspraxis keineswegs ein gänzlich determinierter, sondern ein zu „verhandelnder". Das Verhandeln der eigenen Positionierung trifft dabei auf Grenzlinien und Widersprüche:

- In der Entwicklungsphase konstituierte sich das Internet mit einer männlichen Bedeutungszuschreibung, diese ändert sich aber im Zuge der Kommerzialisierung, sodass eine „benutzerfreundliche“ Oberfläche eine Zuschreibung in Richtung weiblich codiertem Anwendungsbereich eröffnet. Unabhängig von einer tatsächlich geschlechterdifferenten Nutzung bleibt die geschlechtliche Markierung des Internet durch die veränderte Zuschreibungspraxis erhalten.

- Zum Zweiten erfolgt durch die Einführung des Internet als ein primär berufliches Kommunikationsmittel eine Grenzziehung zwischen Berufstätigen und Nicht-Berufstätigen, also zwischen dem geschlechtlich codierten Berufs-/Öffentlichkeitsbe- 
reich und Privatbereich, trägt aber gleichzeitig mit den unterschiedlichen Anwendungsmöglichkeiten zu einer Verwischung der geschlechtlich codierten Grenzen bei.

- Und zum Dritten zieht das Internet in seiner frühen Verbreitungsphase vorerst eine Grenzlinie zwischen höher qualifizierten und niedrig qualifizierten Berufen. Damit trifft die neue Technologie mit einem geschlechtlich segmentierten Arbeitsmarkt zusammen, der sowohl eine horizontale als auch eine vertikale Segmentation aufweist.

Wir haben es hier also mit einem Zusammentreffen von zum Teil gegensätzlichen Bedingungen zu tun, die einen Rahmen für ein geschlechtliches Handeln vorgeben. Die geschlechtlich codierten Bedeutungszuweisungen können nun im Prozess der Anwendungspraxis bestätigt bzw. zurückgewiesen werden. Wie sich dieser Prozess der Geschlechterpositionierung vollzieht, sollte in einer qualitativen Studie näher untersucht werden, wobei ich mich im Folgenden auf einen Teilbereich, der die Befragung von Multimedia-Expertinnen betrifft, beschränke. ${ }^{4}$

\section{Internet als Beruf: eine Expertinnen-Befragung}

\subsection{Methode und Basisdaten}

Um die Prozesshaftigkeit der Geschlechterpositionierung in der beruflichen Alltagspraxis adäquat erfassen zu können, wurden qualitative Interviews mit Internet-Expertinnen geführt. Ziel dieser Befragung war es weniger, die bereits hinlänglich bekannten diskriminierenden Faktoren im Zugang von Frauen zum Internet zu erheben, als den prozesshaften Vorgang sichtbarer zu machen, bei dem die Beziehung von Internet und Geschlecht immer wieder systematisch als geschlechterhierarchische Polarität in der Aneignungs- und Anwendungspraxis hergestellt und mit geschlechterdifferenten Mythen aufgeladen wird.

Befragt wurden insgesamt 23 weibliche Internet-Expertinnen in Österreich. Als Auswahlkriterien galten neben dem Geschlecht die Zugehörigkeit zum Kernbereich, zur ersten oder zweiten Peripherie des Multimediasektors ${ }^{5}$, mit der gleichzeitigen Einschränkung auf den Produktionsbereich und der weiteren Einschränkung auf das Internet (Oberflächenproduktion und Programmierung). Mit dieser Vorgabe sollte die Befragung zum einen auf den Internetproduktionsbereich eingeschränkt, gleichzeitig aber auf Medienverlage und große Unternehmen ausgeweitet werden, um so ein möglichst differenziertes Bild des „doing gender“ in der produktionsbezogenen Alltagspraxis zu erhalten. Die Interviewpartnerinnen waren Programmiererinnen, Webdesignerinnen, Projektleiterinnen und Konzeptionistinnen für Internetprojekte sowie Systembetreiberinnen. Die Auswahl erfolgte mittels Schneeball-Verfahren, ein Verfahren, das sich in-

4 Die Untersuchung wurde im Rahmen eines vom österreichischen BM für Wissenschaft und Verkehr geförderten und von Roman Hummel geleiteten Forschungsprojekts durchgeführt. Zu den weiteren Ergebnissen der Teilstudie „Geschlechterkonstruktion und Internet“ vgl. Dorer (2000 und 2001).

5 Zur Multimedia-Kernbranche zählen nach Michel/Goertz (1999, $20 \mathrm{f}$ und 110) in Agenturen tätige Hersteller/innen von Multimediaprodukten, zur ersten Peripherie Produzent/innen sowie Anwender/innen in Medien-Unternehmen und zur zweiten Peripherie Anwender/innen in sämtlichen Wirtschaftsbereichen. 
sofern anbot, als die Berufsbranche für Multimedia/Internet insbesondere für weibliche Berufstätige noch sehr klein und überschaubar war ${ }^{6}$ und die in diesem Sektor Tätigen voneinander Kenntnis hatten.

Die Befragung wurde von Oktober 1998 bis Januar 1999 mit Hilfe eines teilstandardisierten Leitfadens durchgeführt. Die rund eine Stunde dauernden Gespräche wurden aufgezeichnet und transkribiert. ${ }^{7}$ Die Auswertung erfolgte zum einen nach den üblichen Verfahren der Leitfadenanalyse (Schmidt 2000), zum anderen wurde insbesondere die Sprache als geschlechterkonstituierendes Moment in die Analyse miteinbezogen. Die Sprache gilt damit als Ausdruck geschlechtlicher Selbstrepräsentation, welche auf einen Geschlechterdiskurs verweist, in dessen Rahmen die Befragten sich selbst in der Beziehung von Internet und Geschlecht positionieren. Mit diesem dekonstruktivistischen $\mathrm{Zu}$ gang wird der sprachliche Diskurs nicht primär in seiner Individualität, sondern in seiner gesellschaftlichen Verortung analysiert. Das bedeutet, dass die individuellen Erzählungen gesellschaftliche Diskurse transportieren, die unterschiedlich wahrgenommen, mit Bedeutungen versehen und in das eigene Alltagshandeln integriert werden. (Haug 1995, 1999)

Bezüglich des Alters wurde eine breite Streuung angestrebt. Dennoch zeigt sich, dass es sich bezüglich des Internet um einen jungen Berufszweig handelt. Die Befragten sind zwischen 23 und 43 Jahre alt, sieben sind unter 30 Jahre, 13 zwischen 30 und 39, und drei sind 40 Jahre oder älter (durchschnittlich 32,5 Jahre). Die meisten Befragten sind kinderlos, nur fünf haben diesbezügliche Versorgungspflichten. Von den 23 befragten Expertinnen arbeiten sechs in ihrem eigenen Internet/Multimedia-Unternehmen, ebenso viele für eine Onlinezeitschrift oder einen Verlag, fünf arbeiten in Internetprojekten für staatsnahe Einrichtungen und je drei für nationale/multinationale Konzerne und für kleinere Internet-Betriebe.

\subsection{Ausbildung}

Die befragten Internet-Expertinnen verfügen alle über eine überdurchschnittlich hohe Ausbildung. Zehn haben als höchsten Bildungsabschluss ein absolviertes Studium, acht studieren noch und zwei haben ihr Studium vorzeitig abgebrochen, die übrigen drei Befragten haben Abitur bzw. ein technisches Fachabitur. Mehrheitlich ist die Ausbildung geistes- oder sozialwissenschaftlich orientiert, sechs Befragte verfügen aber über eine technische Ausbildung.

6 Nach einer ersten Erhebung dürfte es im Jahr 1997 rund 90 Multimedia-Produktionsbetriebe in Österreich gegeben haben. Es handelt sich dabei um Klein- und Kleinstbetriebe, die selten mehr als fünf Mitarbeiter haben. Bei der in diesem Kontext durchgeführten Untersuchung zum Berufsbild eines Multimedia-Experten wurden nur männliche Experten befragt. (Hummel / Götzenbrucker 1997, 195) Da es für Österreich keine repräsentative Studie gibt, ist auch der Frauenanteil in dieser Branche nicht bekannt. Für Deutschland haben Michel/Goertz $(1999,122)$ für das Jahr 1998 einen Frauenanteil von 21 Prozent im Multimedia-Kernbereich erhoben, davon war die Hälfte in der Projektleitung, die andere Hälfte in den Bereichen Design und Konzeption tätig, keine einzige Befragte war Programmiererin.

7 Die Interviews wurden von Mag. Gertraud Kohlbacher durchgeführt. Im Zuge der Auswertung wurde eine Anonymisierung und anhand eines Codierleitfadens eine Systematisierung des qualitativen Datenmaterials vorgenommen. Für die Zitate im vorliegenden Text gelten folgende Transkriptregeln: ein Bindestrich markiert eine von der Interviewten gemachte Pause. Punkte ... stehen für eine von mir vorgenommene Auslassung. 
Die Befragten sehen trotz ihrer eigenen - vorwiegend nicht technischen - Ausbildung eine technische Qualifikation als besonderen Vorteil in der Internet/Multimediabranche. Die Hindernisse für eine technische Ausbildung liegen aber nach wie vor in den sozialen Kontexten der Ausbildungszeit sowie den Erfahrungen in männerdominierten Schul- und Studienzweigen - wie in den technischen Schulen mit Abiturabschluss und technischen Studiengängen -, welche für Frauen eine Ausbildungssituation schaffen, die für sie den Status, etwas Besonderes zu sein, abseits der Norm zu sein, stets gegenwärtig sein lassen.

Alle Befragten, die eine technikorientierte Ausbildung absolvierten, berichten von negativen Erfahrungen. Immer wieder mussten sie ihr Interesse für einen „männlichen Bereich“ erklären und rechtfertigen und waren mehr oder weniger massiven Angriffen seitens der Mitschüler und Lehrer ausgesetzt. Das Interesse für Technik als männlich besetztem Raum fungiert als geschlechtlich wirksame Disziplinarstrategie, mit der die geschlechterdifferente Norm, was als „männliches“ und „weibliches“ Interesse zu gelten hat, gefestigt wird. Für Schülerinnen/Studentinnen technischer Ausbildungseinrichtungen bedeutet das Hinwegsetzen über geschlechterdifferent codiertes Interesse stets die Erfahrung der Grenzüberschreitung, der Normverletzung, weil sie als „Andere“ abseits der Norm stehen. Diese Erfahrung ist ebenso wie die Norm diskursiv bestimmt, aber nichtsdestoweniger in ihrer Wirkung real. Das heißt, dass Gefühle des AusgeschlossenWerdens, Gefühle der Andersheit und des Besonderen zwar gesellschaftlich produziert werden, dann aber individuell bewältigt werden müssen.

In dieser Ausbildungssituation bedeutet die gesellschaftliche Norm (Interesse an Technik als männliches Interesse) für Frauen gleichzeitig ein Changieren zwischen männlichen und weiblichen Subjektpositionen. In vielen Fällen wird diese Situation, die ja immerhin mehrere Jahre angedauert hatte, vorwiegend in projektiver Vorgehensweise (erinnert werden Diskriminierungen, die Kolleginnen widerfahren sind) verarbeitet und die eigene Subjektposition als eine eher männliche beschrieben, als ein standhaftes Durchhalten und Durchziehen.

Neben einer Reformulierung und Bestätigung der Norm erfolgte seitens der Befragten immer wieder ein Versuch der Grenzverschiebung bezüglich dieser Norm. Dies wird zum Beispiel klar, wenn eine Befragte die Differenzierung von „harten“ und „weichen" Studienfächern in ihrer geschlechterdifferenten Codierung zwar übernimmt, dann aber Einschränkungen und Differenzierungen vornimmt.

„Elektrotechnik ist noch einmal etwas anderes. Ist halt noch männlicher, ... bei der EDV, da sagt man, das ist wirklich nicht so total typisch männlich. Aber Elektrotechnik, das ist Starkstrom, Nachrichtentechnik wäre Schwachstrom. Das ist schon ein bisschen anders. Aber Elektrotechnik-." (P. K.)

In unterschiedlicher Weise wird in diesem Sinne an der geschlechtlichen Codierung von Technik als männlichem Bereich ständig gearbeitet: Indem Frauen eine technische Ausbildung wählen, überschreiten sie die geschlechtlich markierte Verbindung „TechnikMännlichkeit“, bzw. stellen diese zumindest in Frage. Dann gibt es zahlreiche Momente, wo sie an der Festigung der Verbindung und damit an der Grenzziehung aktiv mitarbeiten, während sie andererseits etwa durch eine weitere Differenzierung von Technik dazu beitragen, dass es zu einer Bedeutungsverschiebung von Technik und damit zu einer Grenzverschiebung kommen kann. 


\subsection{Berufliche Situation}

Die für den Internetberuf nötigen Kompetenzen haben sich die Befragten im Laufe ihrer Berufskarriere meist auf „Umwegen“ angeeignet, entweder in speziellen Kursen, oder als „learning by doing“ in EDV-Abteilungen, Rechenzentralen und ähnlichen Abteilungen. Für die meisten war die Aneignung dieser zusätzlichen Kompetenzen stark interessegeleitet und durch persönliches Engagement gekennzeichnet. Vor allem jene mit einer technischen Ausbildung haben ihre berufliche Karriere sehr zielstrebig in Richtung Internet/Multimedia verfolgt.

Verfolgt man die unterschiedlichen Berufskarrieren der Befragten, so lassen sich die zuvor ausgeübten Tätigkeiten grob in drei Schwerpunktbereiche teilen, die in einem Verhältnis von 3:3:1 stehen:

- Als wichtigste Einstiegstätigkeit können die journalistischen Berufe gelten, die zum einen den klassischen Journalismus, zum anderen Public Relations, Grafik und Verlagswesen umfassen. Die Arbeit mit dem Computer führte für viele rasch in Richtung Online-Journalismus oder Online-Kommunikation.

- Als ebenso wichtiger Bereich ist der EDV-Bereich und EDV-Ausbildungsbereich zu nennen. Im Zentrum steht nicht nur die Technik, in Form von Programmieren und Programmentwicklung, Hard- und Softwaretätigkeiten, sondern auch die Lehrtätigkeit in Form von EDV- und später auch Internetkursen.

- Als dritter bedeutender Bereich kristallisiert sich der Wirtschaftsbereich mit Marketing, Werbung und Finanzen heraus. Werbung und Verkauf über das Internet werden als zukünftige Wachstumsbranchen gesehen (Zimmer 1998, 501), und der Wechsel in die Internet-Branche war oft eine nahe liegende Erweiterung des eigenen beruflichen Tätigkeitsbereichs.

Obgleich die befragten Internet-Expertinnen über ein ausgesprochen hohes Ausbildungsniveau sowie entsprechende Zusatzqualifikationen verfügen, gibt es eine recht eindeutige Verteilung in Bezug auf die zum Zeitpunkt der Befragung ausgeübten Tätigkeitsbereiche. Eine hohe Konzentration finden wir bei jenen Tätigkeitsfeldern, die nicht dem technischen Bereich zuzuordnen sind. Auch die befragten Expertinnen, die eine technische Ausbildung absolviert haben, sind nicht notwendig in der Programmierung, Softwareentwicklung oder Systembetreuung tätig.

Die drei Hauptaufgabengebiete sind Erstellung und Betreunng von Webseiten, die Projektleitung, darunter ist nach Michel und Goertz (1999, 20ff) die verantwortliche Leitung, Koordination und Kontrolle des gesamten Planungs- und Produktionsprozesses zu verstehen, und drittens der Schulungs- und Ausbildungsbereich. Bedeutend weniger häufig nennen die Expertinnen Arbeitsbereiche wie Technik- und Systembetreuung oder die Programmierung und Softwareentwicklung als ihre Aufgabengebiete. Die ebenfalls genannten Aufgabengebiete Beratung und Kundenbetreuung sind im InternetBerufsbereich insgesamt noch unterrepräsentiert, werden jedoch mit zunehmender Konkurrenz in Zukunft an Bedeutung gewinnen. Wenige Nennungen gab es bezüglich des Aufgabenbereichs Betreunng von Chatrooms, einem Bereich, der sich hauptsächlich auf inhaltliche Aspekte (Moderation von Diskussionen, Kontrolle der Einhaltung der Chat-Regeln) nicht aber auch auf technische Aufgaben bezieht. 
Übersicht 1: Tätigkeitsbereiche der Internetexpertinnen (Mebrfachnennungen)

\begin{tabular}{llr}
\hline & Aufgabengebiete & Anzahl der Nennungen \\
\hline 1. & Erstellung und Gestaltung von Webseiten & 15 \\
2. & Projektleitung & 12 \\
3. & Schulungen und Ausbildung & 11 \\
4. & Technik- und Systembetreuung & 5 \\
5. & Beratung, Akquisition und Kundenbetreuung & 4 \\
6. & Programmierung und Softwareentwicklung & 2 \\
7. & Betreuung von Chatrooms & 2 \\
\hline
\end{tabular}

Vor allem drei Punkte fallen bei genauerer Betrachtung der Berufslaufbahn und des aktuell ausgeübten Tätigkeitsbereichs auf, berücksichtigt man zusätzlich die für diesen Kontext relevanten Interviewpassagen der Expertinnen in Bezug auf deren Einschätzung zur Zukunft des Multimedia- und Internetmarktes:

- Zum Ersten zeichnet sich ein Trend ab, der in Richtung Segmentation des Multimedia-Arbeitsmarkts geht. Die meisten Expertinnen sind eben nicht im technischen Bereich und der Programmierung tätig, sondern in der Oberflächengestaltung. Diese und html-Programmierung stellen in der Multimediabranche die neuen Platzzuweisungen für Frauen dar, während die Hardware und das Programmieren als weiterhin gesellschaftlich höher bewertete Platzzuweisung für das Männliche gilt.

- Zweitens zeigt sich, dass eine hohe technische Kompetenz nicht notwendig adäquat beruflich umgesetzt wird, sondern im Gegenteil gesellschaftlich und ökonomisch weniger hoch bewertete Aufgabenbereiche übernommen werden.

- Und drittens zeigt sich, dass Frauen im Multimedia/Internetsektor in einem hohen Maße selbst in der Aus- und Weiterbildung - insbesondere für Frauen als Zielgruppe - tätig sind. Einige von ihnen sind in den von der EU geförderten Projekte im Rahmen von NOW (New Opportunities for Women) tätig und sehen eine wichtige Aufgabe darin, andere Frauen für dieses expandierende Berufsfeld auszubilden und als weibliche Vorbilder zu fungieren.

Was das Einkommen der Internet-Expertinnen betrifft, so variiert dieses beträchtlich (zwischen 1.100 und 3.200 Euro brutto mtl., 14 mal), ist aber gemessen am hohen Ausbildungsstand als eher niedrig einzustufen. Die meisten Expertinnen - insbesondere diejenigen, die eine langjährige qualifizierte Berufserfahrung aufweisen und mehr Einblick in das Einkommensniveau der Berufsbranche haben - schätzen sich als deutlich unterbezahlt ein. Tendenziell lässt sich festhalten, dass das Einkommen in nationalen und multinationalen Konzernen deutlich besser sein dürfte, während es bei Verlagen, im eigenen Unternehmen, in feministisch orientierten Internetprojekten und Multimediabetrieben eher niedrig ist. ${ }^{8}$

8 Das Einkommen ist im Vergleich zu Deutschland, wo Michel/Goertz $(1999,123 f)$ ein monatliches Bruttoeinkommen zwischen $750 \mathrm{DM}$ und $15.000 \mathrm{DM}$ erhoben haben, deutlich geringer. Der Einkommensunterschied zwischen den Geschlechtern liegt in Deutschland bei 1.000 DM brutto monatlich, für Österreich gibt es für die Multimediabranche keine Vergleichszahlen. Nach der letzten Erhebung des österreichischen statistischen Zentralamts liegt für 1999 aber der durchschnittliche Einkommensunterschied alle Berufszweige betreffend bei 35 Prozent. 


\subsection{Interneteinstieg und Selbstrepräsentation}

Von den befragten Expertinnen gehören nur fünf zu den „Pionierinnen“, die bereits Anfang der neunziger Jahre mit dem Internet arbeiteten. (Definition nach Eimerer et al., 1998, 425) Die Mehrheit der von uns Befragten gehört nicht zu den „Pionierinnen“, sondern können als so genannte „Früheinsteigerinnen“ bezeichnet werden. Der Ersteinstieg erfolgt also zu einem Zeitpunkt, als das Internet erstens mit dem Netscape bzw. dem Internet Explorer bereits eine benutzerfreundliche Oberflächengestaltung aufwies und zweitens im beruflichen bzw. betrieblichen Umfeld eine größere Expansion erfuhr.

Die ersten Erfahrungen mit dem Internet wurden von den Befragten fast ausschließlich im beruflichen Bereich und zum Teil gemeinsam mit anderen gemacht. In der Erinnerung an die Einstiegssituation sind die anderen Personen zuerst einmal männlich. Es sind die Kollegen im Büro, die jungen Mitarbeiter in der Firma, es sind die Kursteilnehmer, die beim Ersteinstieg dabei waren. Interessant ist, dass erst bei einem konkreten Nachfragen nach dem Geschlecht der anderen auch Frauen in der Einstiegssituation eine wichtige Rolle gespielt haben und so die Auslassung von Frauen durch Frauen hergestellt wird. Nur zwei Befragte gaben explizit eine weibliche Person an, mit der sie gemeinsam die erste Netzerfahrung erworben haben. Wie auch immer die Einstiegssituation in Wirklichkeit gewesen sein mag, in der Erinnerung der Befragten verläuft offenbar zuerst einmal die gedankliche Zuschreibung über die Koppelung von „Technik und Männlichkeit“. In diesen Erinnerungsszenarien zeigt sich, dass die Verbindung Geschlecht und Technik auf sehr subtile Weise wirksam wird. Denn die Assoziationskette: „Männlichkeit - Technik - Unterstützung/Hilfe“ wird all zu schnell hergestellt. Mit dieser Assoziation wird die vorherrschende Vorstellung, Frauen würden bei neuen technischen Medien männliche Hilfestellungen benötigen, repräsentiert und bestätigt.

Entscheidend bei dieser Selbstrepräsentation ist, dass die befragten Expertinnen sehr wohl ihr Interesse, ihre eigenen Aktivitäten, sich die neue Technologie anzueignen, betonen, und trotzdem wird auf die genannte Assoziationskette: „Männlichkeit - Technik - Unterstützung/Hilfe“ nicht verzichtet. Im nachfolgenden Beispiel etwa erzählt die Befragte sehr selbstbewusst - aus einer quasi männlichen Subjektposition - von ihrem Ersteinstieg im Büro (wo Frauen und Männer arbeiteten, was aber im Gespräch nicht erwähnt wurde). Diese Selbstpositionierung wird wieder relativiert, indem dann eben doch der Freund als die treibende Kraft für das weitere Computerinteresse ins Gespräch eingebracht wird. Wie widersprüchlich hier die Selbstkonstruktion erfolgt, zeigt sich an der Art, wie der Freund sprachlich in das Gespräch hereingeholt wird. Die Formulierung „Also, wo halt-ja-zum Teil ... “ macht deutlich, dass die ambivalente Selbstkonstruktion nicht ganz konfliktfrei funktioniert.

„Also das war hier, im Büro. Da hat es Internetzugang gegeben. ... Also ich hab eine Affinität zu Computern, ich mag die Dinger und-ja ich habe immer mehr geschaut, was es da gibt, und irgendwann habe ich dann Webseiten machen gelernt. Also, wo balt-ja-zum Teil meine Beziehung halt zu meinem Freund auch mit Schuld ist, weil er eben Informatik studiert und er sich unbeimlich gefreut hat, dass er mal eine Freundin hat, die sich dafür interessiert. "(Z. A.)

Auf der anderen Seite gibt es auch Selbstpositionierungen, die nur das eigene Interesse und die Begeisterung für das Neue betonen. In fünf Erzählungen erfolgt der Einstieg durch eigenen Antrieb und ohne Einstiegshilfe: 
„Ich weiß es nicht mehr genau, aber ich bin im Computerzentrum gesessen, ... da habe ich alle Programme durchprobiert, habe überall draufgedrückt und irgendwo hat es dann Mosaic gegeben, das war der Vorläufer von Netscape. ... Und dann machst du das ganz intuitiv, dass du da draufklickst." (V. B.)

Insgesamt verlaufen die meisten Erzählungen über die eigene Einstiegserfahrung mehr oder weniger entlang des vorherrschenden Geschlechterdiskurses. Die Verbindung nicht zwingender, aber dennoch als fast natürlich erscheinender Bereiche bzw. die „Artikulation“ im Sinne der Cultural Studies (Hall 1996a, 33, Hall 1996b, 141ff) von „Technik - Männlichkeit“ wird bei den Erinnerungen selten hinterfragt. Dass diese aber dennoch nicht mehr einwandfrei funktioniert, zeigen die unterschiedlichen, teils recht ambivalenten Annäherungen, sei es etwa in der Betonung der eigenen Interessen und Motivationen, oder in der gebrochenen Form, wie die Artikulation von „Männlichkeit und Technik" in der Erinnerung thematisiert wird. In Summe bleibt jedoch festzuhalten, dass auch die befragten Expertinnen, die schon aus beruflichen Gründen einen stärker motivierten Umgang mit der neuen Technologie haben, in oben gezeigter Weise an der Konstruktion und Aufrechterhaltung eines Technikmythos, der mit einer Affinität zum Männlichen einhergeht, in unterschiedlicher Weise beteiligt sind.

\subsection{Selbstrepräsentation im Beruf}

Auch bei der Selbstrepräsentation im Beruf wird deutlich, dass wir es nicht mit einer einzigen „weiblichen“ Subjektposition zu tun haben, sondern dass es auch hier sowohl Affirmationen als auch Versuche der Grenzverschiebung innerhalb des geschlechtlich codierten Technikdiskurses gibt.

Mit der vermehrten Beteiligung von Frauen in Internet/Multimedia-Berufen zeichnet sich zuerst einmal kein Aufbrechen der Geschlechterbinarität ab, sondern eine Differenzierung des Technikdiskurses. Oberflächengestaltung oder html-Programmierung scheinen die neuen Platzzuweisungen für Frauen zu werden, während die Hardware und das Programmieren weiterhin als gesellschaftlich höher bewertete Platzzuweisung für das Männliche gilt. Wie sehr diese Aufwertung des technischen Bereichs und die Abwertung von Arbeiten an der Oberfläche miteinander verbunden werden, spricht eine Befragte ganz beiläufig an:

„Das (html-Programmieren) war für mich durchschaubar. Das ist für mich - ah, das begreif ich, was beim html-Programmieren abläuft. Und für Hardcore-Programmierer ist html sicher irgendwie so eine - eine Art Kinderübung, ... (Q.J).

Die Verbindung „Technik - Männlichkeit - Hardcore“ wird in einer beiläufigen Bemerkung in einer so selbstverständlichen Weise vorgenommen, die mehr als erstaunlich ist. Auch die Verbindung „Frau - btml-Programmierung - Kinderübung “ verstärkt nicht nur die Differenz, sondern auch den Abstand zwischen Aufgewertetem und Abgewertetem. Sichtbar wird, wie automatisch die Dichotomisierung erfolgt und wie wirksam gesellschaftliche Geschlechterzuschreibungen dabei sind. Das eigene Vordringen in einen „nicht-weiblichen“ Bereich wird nicht mit Stolz als Normüberschreitung interpretiert, sondern mit einer Grenzverschiebung beantwortet, indem die htmlProgrammierung eine weibliche Codierung erhält und gleichzeitig in unverhältnismäßiger Weise abgewertet wird. Über die Konstruktion einer neuen Grenze - zwischen dem „richtigen“ Programmieren und der html-Programmierung - und der damit 
verbundenen Bewertung erhält die „männliche“ Technik die ursprüngliche Wertigkeit zurück.

Entgegen der Vermutung, Pionierinnen der neuen Technologien würden männlich codierte Felder aufbrechen, zeigt sich, dass geschlechterdifferente Markierungen neu gesetzt werden und zwar in einer Weise, die wenig bis kaum von traditionellen Formen der Platzzuweisung mit den ihnen zugeschriebenen Wertigkeiten (wie höheres und niedrigeres Prestige) abweichen. Eine Rolle bei dieser Platzzuweisung spielen nicht nur jene gesellschaftlichen Strukturen, die sich in historischen Prozessen herausgebildet haben, sondern auch die konkreten Situationen, in denen Männer und Frauen ihr Einverständnis mit dieser geschlechterdifferenten Norm abgeben und den Umgang mit der neuen Technologie in gesellschaftliche Praxis umsetzen. Es handelt sich dabei keineswegs um eine glatte, widerspruchslose geschlechtliche Subjektsetzung, vielmehr zeigen die Aussagen in den Interviews, dass die Selbstrepräsentation durch jene Ambivalenz gekennzeichnet ist, die sich letztlich nicht eindeutig einer einzigen geschlechtlichen Positionierung zuordnen lassen. Die als männlich und weiblich codierten Eigenschaften sind jeweils Bestandteile einer einzelnen Selbstpräsentation, wobei immer wieder die gesellschaftlichen Normen für eine weibliche Subjektsetzung die Leitlinie für die eigene geschlechtliche Positionierung abgeben.

Neben einer typisch „weiblichen“ Positionierung, die sich in der Abwertung oder aber in der Geringschätzung des eigenen Aufgabengebietes ausdrückt, finden wir auf der anderen Seite häufig Selbstrepräsentationen, die eine selbstbewusste Bewertung der eigenen Arbeit und eine Selbstverständlichkeit erkennen lassen, mit Technik und technischen Fragen umzugehen, wie sie gesellschaftlich von Frauen nicht erwartet wird.

„Mein Firmenpartner und ich treten auf und unlängst ist uns das passiert: Er ist der Kommunikationsfuzzi, komplett auf Softskills, keine Abnung von Computer, ich bin die Computertante. Wir sitzen vor zwei Leuten, die sich über unser Angebot unterhalten. I hn fragen sie die technischen Fragen und mich fragen sie die Fragen nach der sozialen Kompetenz, und dann wird kreuzgeantwortet. Dann schauen sie einmal blöd, aber dann geht es. ... Mir wär' es manchmal schon recht, wenn ich ganz normal arbeiten könnte. "(X. C.)

Immer wieder mit dem Geschlecht konfrontiert zu werden, ist ärgerlich. Der Ärger bezieht sich dabei auf die Personen und ihr Verhalten, die diese geschlechtliche Position in Frage stellen. In dieser Konfrontation zeigt sich, wie mühsam es ist, von gesellschaftlich vorgegebenen Geschlechterpositionen abzuweichen, und zuvor erst diese geschlechtlichen Zuschreibungen zurückweisen zu müssen, um anschließend die Fachkompetenz, die männlich codiert ist, unter Beweis stellen zu können. Fachkompetenz und Professionalität im Multimediaberuf erhalten so eine geschlechtliche Codierung, indem sie mit Männlichkeit gleichgesetzt werden. Frauen wird diese zuerst einmal abgesprochen, sie werden zuerst einmal auf die gesellschaftliche Erwartung, was Weiblichkeit zu sein hat, fixiert.

Die immer wieder aufs Neue notwendige Konfrontation und Klarstellung bezüglich Fachkompetenz und beruflicher Professionalität lässt damit das Hin- und Herbewegen zwischen den geschlechtlichen Positionen zugleich zu einem mühsamen Prozess der eigenen Identitätskonstruktion werden. Der Effekt ist der, dass in diesem Fall entweder immer aufs Neue die Konfrontation und Klarstellung erfolgen muss, oder aber diese geschlechterdifferente grenzüberschreitende Position zugunsten gesellschaftlich vorgegebener Geschlechterpositionen aufgegeben wird. 


\subsection{Karrierepläne und Zukunft}

Die gesellschaftliche Zuschreibung, Frauen würden zugunsten einer eigenen Familie auf ihre Karriere verzichten, ist ebenso weit verbreitet wie die Vorstellung, Frauen würden einen Karriereaufstieg gar nicht anstreben. Dieser häufig erhobene Befund verstellt den Blick auf die geschlechterdifferenten Strukturen mit ihrer unsichtbaren Karriere-Grenze für Frauen, welche ihren manifesten Ausdruck in der Metapher der „gläsernen Decke“ finden. Dieser gesellschaftliche Aspekt ist gerade bei einem Berufszweig wie der Multimediabranche von besonderem Interesse, weil er noch als eine männliche Domäne gilt. Aber nur wenige Befragte meinen, dass sie keine Top-Karriere anstreben würden, sie würden lieber ihre Tätigkeit solange ausüben, solange es ihnen Spaß macht. Diese Befragten thematisieren bei ihren Karrierevorstellungen auch die Familienplanung. Bei diesen Aussagen finden wir eine Subjektpositionierung, die jenen Weiblichkeitsdiskurs in einer Weise abändert, der nicht den Verzicht zugunsten der Familie in den Vordergrund rückt, sondern den Wunsch, eine sinnvolle Tätigkeit, eine Tätigkeit, die Freude bereitet, auszuüben. Obgleich es hier nicht mehr um Verzicht, sondern um eine positive Erweiterung geht, lassen sich diese Aussagen einem Weiblichkeitsdiskurs zuordnen, der sich weniger inhaltlich als in seiner Rhetorik verändert haben dürfte.

Wenn wir uns die Antworten auf die Frage nach den Zukunftsplänen der Befragten ansehen, so fällt auf, dass mehrere Befragte ihre Aussagen mit Formulierungen beginnen, wie "Hm, ich weiß noch nicht genau", "Ich weiß nicht, ich überlege mir", "Ich weiß es nicht wirklich“, „Ah, ich weiß meinen Weg nur ungefähr". In diesen Satzeinleitungen drückt sich jenes diskursive Moment aus, das Frauen den Platz in unteren hierarchischen Ebenen zuordnet. Offensichtlich werden mit diesen Formulierungen gesellschaftliche Erwartungen zum Ausdruck gebracht, die keineswegs die individuelle Meinung wiedergeben müssen. Denn im weiteren Verlauf der Gespräche formulieren die meisten Frauen sehr konkrete berufliche Ziele bzw. berufliche Etappen, die sie vor sich sehen und die sie erreichen möchten. Die meisten Befragten haben konkrete Karrierepläne, und verweisen darauf, dass sie ihre berufliche Zukunft nicht dem Zufall überlassen möchten. Die eingangs gemachten relativierenden Aussagen stehen damit im Widerspruch zu ihren weiteren Ausführungen und verweisen darauf, wie schwierig es ist, Sprachmuster, die dem Weiblichen zugeordnet werden, aufzubrechen.

Die Zukunftspläne sind dabei sehr unterschiedlich. Mehrere sprechen die Zukunft des Internet bzw. der neuen Technologien an und meinen, dass dieses Berufsfeld nicht unbedingt die Erwartungen bezüglich Arbeitsplätzen und Einkommen erfüllen werden. Vielfach wird auch erkannt, dass die Multimediaproduktion geschlechterdifferente Arbeitsplätze zur Verfügung stellen wird, dass die Erstellung einer Homepage oder die Erstellung von Webseiten vermutlich nicht jener Arbeitsbereich sein wird, der zu den gut bezahlten Jobs der Multimediabranche zählen wird.

„Ich habe die html-Produktion übernommen und habe mir gedacht, dass ich da auch mehr Geld bekommen und irgendwie auch mebr Mitsprache bekomme, aber das hat sich alles als Luftschloss erwiesen" (O. L.)

Andere wiederum möchten ihre Tätigkeit in Richtung ganzheitlicher Tätigkeit verändern, einen Schritt weg von fachlich einschlägigen Arbeiten in Richtung Gesamtschau von Technik und Inhalten vornehmen. 
"Ich habe eine technische Ausbildung und was mir bisher gut gefallen hat, ist, dass ich nicht nur Technik mache, dass ich nicht nur diese Fachidiotenschiene mache, nur Technik und sonst nichts. ... An und für sich möchte ich meine jetzige Richtung weitermachen. Einfach die Verbindung von meiner Ausbildung und Frauenprojektarbeit." (S. H.)

Interessant ist auch, wie von den Expertinnen die männlich codierte Technik, die mit den gesellschaftlichen Zuschreibungen von bedeutsam, gewichtig, prestigeträchtig, hohem Einkommen etc. gekoppelt ist, abgewertet wird. Die nicht dem gesellschaftlichen Diskurs über Technik entsprechende Bewertung auf Basis der eigenen technischen Ausbildung erhält eine kritische Komponente, indem die Kontexte von Technik als wesentliches Moment für die Aneignung und Praxis von Technik interpretiert und damit auch die gesellschaftlichen Auswirkungen miteinbezogen werden.

Viele Befragte sprechen von einer Zukunft, in der sie ihr eigenes Unternehmen führen möchten bzw. ihr eigenes Unternehmen weiter ausbauen wollen. Dies entspricht durchaus einer Entwicklung, die sich im Multimediabereich abzeichnet. Derzeit gibt es einen Gründungsboom von kleinen Multimediabetrieben, die erst in einigen Jahren einem Marktbereinigungsprozess unterworfen sein werden, wobei nur größere Unternehmen auch tatsächliche Chancen haben werden, diesen Prozess zu überleben.

\subsection{Erfabrungen mit männlicher Dominanz im und rund ums Netz}

Individuelle Erfahrungen und die Erinnerung an diese Erfahrungen implizieren immer auch das Moment einer sozialen Wahrnehmung. Eine soziale Wahrnehmung, die sich im Prozess der historischen Entwicklung herausgebildet hat und Veränderungen unterworfen ist, lässt sich als gesellschaftliches Wahrnehmungsdispositiv beschreiben, das seinen Ausdruck in der öffentlichen Rede ebenso wie in den privaten Erzählungen findet. Wenn wir also nach persönlichen Erfahrungen fragen, sind die gesellschaftlichen Diskurse in diese Erfahrungen eingeschrieben. Erfahrung stellt sich damit weder als ein von der Gesellschaft determiniertes Handlungsdispositiv dar, noch als ein subjektives Handeln oder Verhalten, das nach eigenem Belieben frei wählbar wäre. Etwas über Erfahrungen zu ermitteln, bedeutet also nach Haug $(1995,9)$ immer zugleich mitzudenken, was an gesellschaftlichen Erfahrungen von den Einzelnen wie wahrgenommen, selbsttätig mit Bedeutungen versehen und in die eigene Alltagspraxis eingegliedert wird.

Fast alle Expertinnen erzählen von Erfahrungen mit männlicher Dominanz im Internet. Sie äußern sich dabei vorwiegend über Belästigung bzw. „Anmache“ - wie sie es sehr oft selbst bezeichnen - in Form von anonym erhaltenen E-Mails und anzüglichen Bemerkungen in Newsgroups und in Chats.

Auch die extrem leichte Abrufbarkeit von Sex- und Pornoseiten im Internet wird erwähnt und als unangenehm dargestellt. Die meisten Expertinnen sprechen in einer Art und Weise darüber, als ob diese Dinge im Netz einfach hinzunehmen wären. Die Verbindung von Internet, Männlichkeit, Sex und Pornografie ist eine im öffentlichen Diskurs immer wieder hergestellte. Vor allem die populären Medien sind es, die in der kommerziellen Einführungsphase des Internets prioritär männlich codierte Themen wie Cybersex, Kinderpornografie u.a. aufgegriffen haben bzw. aufgreifen und damit an der geschlechterhierarchischen Positionierung des Internet wesentlich mitarbeiten. Für die Befragten, die über ihre Erfahrungen sprechen, tritt so ein in der öffentlichen Diskussion ausführlich thematisierter Sachverhalt ein, der als Bestätigung dessen gelten kann, was ohnehin jede/r bereits weiß. 
Ein diskursiv produziertes Wissen trifft hier auf persönliche Erfahrung, die dann nicht den Neuigkeitscharakter besitzt, den sie ohne mediale Inszenierung und öffentliche Debatte hätte. Diese „Artikulation“ (Hall 1996a, 1996b), d.h. eine Verbindung, die nicht zwingend, aber dennoch als scheinbar „natürlich“ vorhanden ist oder etabliert wird, wird hier als eine geschlechtlich konnotierte Verbindung hergestellt bzw. rekonstituiert. Dies ist auch der Grund dafür, dass Belästigungen genannter Art nicht wirklich bewusst wahrgenommen werden, oder aber im Zusammenhang mit dem Internet als etwas „Natürliches“ betrachtet und daher oft kommentarlos in Kauf genommen werden. Diese Art der Wahrnehmung hängt aber auch davon ab, in welchen sozialen Kontexten die Befragten leben und arbeiten. So etwa zeigt sich, dass sich jene Expertinnen, die in feministischen Projekten tätig sind oder auf ihren feministischen Hintergrund verweisen, weit expliziter und reflexiver mit der Verbindung Internet - männliche Belästigung auseinander setzen.

Analog verhält es sich bei den Erzählungen rund ums Internet und der Erfahrung, in einem männlich dominierten Berufsfeld tätig zu sein. Hier geht es vor allem um die Verbindung „Technik - Internet und Männlichkeit“, die stets reproduziert wird. Auch hier gibt es unterschiedliche Wahrnehmungsdispositive und unterschiedliche Arten, mit Diskriminierungserfahrungen umzugehen. Diese reichen von Nicht-Wahrnehmen über die Akzeptanz ob der Einsicht in die Unveränderlichkeit, die kritisch-reflexive Distanznahme bis zu energischem Auftreten. Als Handlungsstrategien können mehrere Muster unterschieden werden. Da gibt es jene, die sich an die Männerdomäne und die Diskriminierung gewöhnt haben und diese als Norm akzeptieren, und andere, die sich quasi in einem Einzelkämpferinnentum die nötige Durchsetzungsfähigkeit erworben haben, und wiederum andere, die die Bedingungen nicht akzeptieren und gesellschaftsverändernde Ansprüche stellen. Aber bei allen geht es immer wieder auch darum, Fachkompetenzen und berufliche Professionalität im Multimediabereich ständig unter Beweis stellen zu müssen.

Die eigenen Erfahrungen verbinden sich mit einem diskursiven Wissen, das den Bereich Technik als Männerdomäne zusätzlich mit bestimmten männlich codierten Eigenschaften auffüllt. In ihrer Überdeterminierung erzeugt diese Verbindung jene Effekte, die wir als typische Weiblichkeitsdiskurse kennen. Das ist zum einen der Opferdiskurs, der Frauen in einer typisch weiblichen Subjektposition belässt, in der die Umstände als Norm akzeptiert und hingenommen werden, zum anderen jenes Einzelkämpferinnentum, bei dem Frauen selbstbewusst in ihren Handlungsstrategien Geschlechternormen überschreiten und in einer männlichen Subjektposition offensiv und aggressiv agieren, zum Dritten finden wir jenen feministischen Diskurs, bei dem auf unterschiedliche Weise versucht wird, geschlechtersensitiv gesellschaftsverändernd wirksam zu werden.

\subsection{Chatrooms, MUDs und das Spiel mit Geschlechteridentitäten}

Ein besonderes Interesse der feministischen Forschung in Bezug auf die Netzkommunikation gilt der Frage nach der Möglichkeit der Aufhebung der Geschlechterbinarität. Die Möglichkeit eines anonymen Zugangs, das Zurücktreten des Körpers im Cyberspace und die damit verbundene Beliebigkeit der Selbstrepräsentation lassen Utopien von neuen Identitätskonstruktionen und vom Verschwinden einer geschlechtlichen Identität als zentrale Themen einer neuen Technikdiskussion breiten Raum einnehmen. Chatrooms und MUDs sind dabei jene Räume des Internets, in denen neue Identitäten abseits von Klasse, Ethnie und Geschlecht erprobt werden können. Gender-Swapping (Einloggen als ein anderes Geschlecht), auch manchmal weniger zutreffend als virtuel- 
les Cross-Dressing bezeichnet, erlaubt ein für das reale Leben gefahr- und konsequenzloses Ausprobieren einer anderen geschlechtlichen Subjektposition. Das Verlassen der eigenen Geschlechterkultur in der virtuellen Realität ermöglicht potenziell einen neuen Erfahrungshorizont. Nach Turkle $(1995,1996)$ lassen sich damit geschlechterdifferente Alltagspraxen als Konstruktionen geschlechtlich geformter Denk-, Gefühls- und Handlungsdiskurse erkennen. Mit dem Vertauschen der Geschlechterposition können so gesellschaftliche Zuschreibungspraxen im Spiel erfahren werden, so dass den User/innen in einer Art Selbstreflexion die gesellschaftliche Konstruiertheit der eigenen Geschlechteridentität bewusst werden kann.

Entgegen unseren Erwartungen waren die befragten Expertinnen bezüglich der Frage nach den Erfahrungen mit dem Spiel mit anderen geschlechtlichen Identitäten äußerst zurückhaltend. Fünf Befragte hatten damit noch keine persönliche Erfahrung gemacht. Zum Teil ist es einfach Desinteresse, zum Teil halten sie es für Zeitverschwendung und zum Teil haben sie sich zwar theoretisch mit diesen Möglichkeiten auseinander gesetzt, jedoch noch nicht in der Praxis erprobt. Die übrigen Befragten erzählten ihre mehr oder weniger umfangreichen Erfahrungen mit dem Gender-Swapping, lehnen dieses aber zum Gutteil ab. Für sie ist sowohl Chatten als auch Gender-Swapping uninteressant und nicht wert, die Zeit damit zu „vergeuden“. Einige finden es aber auch ganz „witzig“, das andere Geschlecht zu spielen, ohne sich jedoch über theoretische Implikationen Gedanken zu machen.

Nur sechs Befragte - und zwar nur die jüngeren Expertinnen - haben sich umfangreicher mit der Möglichkeit eines Geschlechterwechsels in Chats und MUDs beschäftigt. Darunter finden sich nicht nur jene Befragten, die selbst Chatrooms betreuen, sondern auch eher jene, die in feministischen Projekten tätig sind. Sie halten die Idee für eine äußerst reizvolle Utopie, meinen aber, dass die Möglichkeiten einer gesellschaftlichen Veränderung in Bezug auf die Geschlechterordnung mittels neuer Technologien weit überschätzt würden. Dennoch sehen sie, wie durch das Zurücktreten des Körpers neue Wahrnehmungsdispositive erzeugt werden können, und dass das Geschlecht ganz offensichtlich einen Stellenwert einnimmt, ohne den Identität nicht vorstellbar ist.

„Du hast sofort ein Bild, wer das sein könnte. ... Also vielleicht nicht wirklich ein Gesicht visualisiert, aber die Person halt, ob das jetzt Frau oder Mann ist." (L. O.)

„Also ich glaub, dass sich relativ viele Männer so virtuelle Frauen erschaffen. Einen, den ich auch sebr gut kenne, der steigt hin und wieder als Frau ein. ... Nimmt halt die Männer da aufs Korn und spielt Frau. Also er hat mir erzäblt, ibm bätte das wirklich viel gebracht. ... Weil er mehr verstanden hat, was es eigentlich heißt, eine Frau zu sein. Weil er irgendwie gemerkt hat, dass es, auch wenn man es nicht so meint, als Typ, irgendwie so blöde Meldungen rübergeschoben bekommt. Aber er hat für sich jetzt entdeckt, dass es doch dann auch die Unterschiede gibt und dass es nicht einfach ist, in so einem Umfeld, eine Frau zu sein. ... Ich meine, die Möglichkeit, sich anonymisiert mit jemanden zu unterhalten, hat natürlich irgendwie einen Einfluss auf dein Leben. Das ist ganz klar. Und wenn du da jetzt noch Geschlechter tauschen kannst, dann ist es ja auch eine logische Schlussfolgerung - dann weißt $d u$ auch vielleicht besser, wie es dem anderen geht. ... Dass man nicht bei jedem Ereignis jetzt super was lernt für das Leben - ist auch klar. Allein der anonyme Zugang dann ist es egal, ob ich Mann oder Frau bin, egal, wer ich bin, wie ich ausschaue." (D. W.) 
Das Spiel mit Geschlechteridentitäten, das Chatten oder das Spiel in MUDs ist für NetzExpertinnen von sehr geringem Interesse. Das liegt zum einen daran, dass sie ohnehin ständig mit dem Netz beruflich zu tun haben, zum anderen auch daran, dass sie in einem beruflichen Umfeld, das derzeit noch männlich dominiert ist, offensichtlich wenig dafür erübrigen können, in Chats oder MUDs männliche Identitäten zu übernehmen.

\section{Fazit}

Das Gender-System als ein symbolisches bzw. System der Bedeutungsproduktion gibt auch für das Internet kulturelle Konzeptionen von Männlichkeit und Weiblichkeit als zwei sich ausschließende Kategorien vor. Sowohl in sozialen Repräsentationen (wie etwa der medialen) als auch in Selbstrepräsentationen zeigt sich, dass Geschlechterpositionen weniger leicht aufzubrechen sind, als es Theorien der Cyberfeministinnen vermuten lassen. Dennoch lassen sich Möglichkeiten der Grenzverschiebung und Neupositionierung erkennen, wenngleich die medialen Repräsentationen der populären Medien noch konservative Geschlechterdifferenzen und -differenzierungen bemühen.

Die qualitative Studie zeigt, dass der Mythos, Internet hätte etwas mit Technik zu tun, die diskursive Praxis des geschlechtlichen Handelns (doing gender) in entscheidendem Maße beeinflusst, doch werden auch Gegenentwürfe sichtbar. Denkräume zu schaffen, kulturelle Erzählungen neu zu schreiben und neue Perspektiven zu entwickeln, gelingt am ehesten aus der Wahrnehmung der Spannung und Widersprüchlichkeit der eigenen Positionierung heraus. Die Verortung in feministischen Kontexten oder Erfahrung in technikorientierten Ausbildungen bietet hier eine Möglichkeit kritischerer Distanznahme.

\section{Literatur}

ARD/ZDF-Arbeitsgruppe Multimedia (1999): Internet - (k)eine Männerdomäne. In: Media Perspektiven, H. 8, S. $423-429$.

Cockburn, Cynthia/Ormrod, Susan (1997): Wie Geschlecht und Technologie in der sozialen Praxis „gemacht“ werden. In: Dölling, Irene/Krais, Beate (Hg.): Ein alltägliches Spiel. Geschlechterkonstruktion in der sozialen Praxis. Frankfurt/M.: Suhrkamp, S. 17 - 47. (engl.: London 1993)

de Lauretis, Teresa (1996): Die Technologie des Geschlechts. In: Scheich, Elvira (Hg.): Vermittelte Weiblichkeit. Feministische Wissenschafts- und Gesellschaftstheorie. Hamburg: Hamburger Edition, S. 57 - 93. (engl.: Bloomington 1987)

Dorer, Johanna (1997): Gendered Net. Ein Forschungsüberblick über den geschlechtsspezifischen Umgang mit neuen Kommunikationstechnologien. In: Rundfunk und Fernsehen, 45. Jg., H. 1, S. $19-29$.

Dorer, Johanna (2000): Geschlechterkonstruktionen in der Aneignung und Anwendung des Internet. Ergebnisse einer qualitativen Studie. In: Medien und Zeit, Jg. 15, H. 2, S. $40-51$.

Dorer, Johanna (2001): Internet und Geschlecht. Berufliche und private Anwendungspraxen der neuen Technologie. In: Klaus, Elisabeth/Röser, Jutta/Wischermann, Ulla (Hg.): Gender Studies und Kommunikationswissenschaft. Opladen: Westdeutscher Verlag. (im Druck)

Eimerer, Birgit/Gerhard, Heinz/Oemichen, Ekkehardt/Schröter, Christian (1998) Onlinemedium gewinnt an Bedeutung. Nutzung von Internet und Onlineangeboten elektronischer Medien in Deutschland. ARD/ZDF-Online-Studie 1998. In: Media Perspektiven, H. 8, S. 423 - 435.

Hall, Stuart (1996a): Signification, Representation, Ideology: Althusser and the Post-Structural Debates. In: Curran, James/Morley, David/Walkerdine, Valerie (Eds.): Cultural Studies and Communication. London/New York/Sydney/Auckland: Arnold, S. 11 - 34.

Hall, Stuart (1996b): On Postmodernism and Articulation. In: Morley, David/Chen, Kuan-Hsing 
(Eds.): Stuart Hall. Critical Dialogues in Cultural Studies. London/New York: Routledge, S. $131-150$.

Haraway, Donna (1990) [1985]): A Manifesto for Cyborgs. Science, Technology, and Socialist Feminism in the 1980s. In: Nicholson, Linda (ed.): Feminism/Postmodernism. London/New York: Routledge, pp. 190-233. (dt.: Frankfurt/M. 1995)

Haug, Frigga (1995): Das Projekt. In: Haug, Frigga/Hipfl, Brigitte: Sündiger Genuss? Filmerfahrungen von Frauen. Hamburg: Argument, S. 7 - 14.

Haug, Frigga (1999): Vorlesungen zur Einführung in die Erinnerungsarbeit. Berlin/Hamburg: Argument.

Herner, Christine (2000): Die Töchter der Madam Curie. In: Wiener Zeitung, Extra 14./15. Juli, S. 12.

Hoffmann, Ute (1987): Welchen Anteil haben Frauen an der Computergeschichte und -arbeit? München: Hampp Verlag.

Hummel, Roman/Götzenbrucker, Gerit (1997): Wenig lernen - alles können. Empirische Ergebnisse zur Entwicklung von Multimediaberufen in Österreich. In: SWS-Rundschau, 37. Jg., H. 2, S. 191-104.

Kroker, Arthur/Weinstein, Michael (1994): Data Trash. The Theory of the Virtual Class. New York: St. Martin's Press. (dt. Wien 1997)

Lovink, Geert/Schultz, Pit (1999): Aus der Schatzkammer der Netzkritik. In: Maresch, Rudolf/ Weber, Niels (Hg.): Kommunikation - Medien - Macht. Frankfurt/M.: Suhrkamp, S. 299-328.

Makivic, Svetlana (2000): Die Internetqueens. http://www.zdf.de/ratgeber/aktuell/monalisa/ 39331/index.html (letzter Zugriff: 22.03.2001)

Michel, Lutz/Goertz, Lutz (1999): Arbeitsmarkt und Multimedia: Trends und Chancen. Qualifikationsprofile und Karrierewege in einer Zukunftsbranche, Berlin: Vistas. Ebenso unter: http://www.mmb-michel.de/mmquali99_kurz.doc (letzter Zugriff: 22.03.2001)

N.N. (2000): Amerikas Frauen holen auf. Online-Spiegel v. 10.8.2000.

ORF (2000): Strukturvergleich -2. Quartal 2000. http://mediaresearch.orf.at/aim/05/05_2q00.htm (letzter Zugriff: 22.03.2001)

Pfeiffer, Carola (2000): Die Schattenseiten der IT-Branche. http://www.zdf.de/ratgeber/aktuell/monalisa/39332/index.html (letzter Zugriff: 22.03.2001)

Plant, Sadie (1997): Zeros and Ones. London: Fourth Estate (dt.: Berlin 1998).

Schmidt, Christiane (2000): Analyse von Leitfadeninterviews. In: Flick, Uwe/Kardorff, Ernst von/Steinke, Ines (Hg.): Qualitative Forschung. Ein Handbuch. Reinbek: Rowohlt, S. 447 455.

Turkle, Sherry (1995): Life on the Screen. Identity in the Age of the Internet. New York/London: Simon \& Schuster. (dt. Reinbek b. Hamburg 1998)

Turkle, Sherry (1996): Parallel Lives: Working on Identity Space. In: Grodin, Debra/Lindlof, Thomas (eds.): Constructing the Self in a Mediated World. London: Sage, pp. 156-178.

Zimmer, Jochen (1998): Werbemedium World Wide Web. Entwicklungsstand und Perspektiven von Onlinewerbung in Deutschland. In: Media Perspektiven, H. 10, S. 498 - 507. 